\title{
PEP-II AND KEKB OPERATIONAL STATUS *
}

\author{
J. Seeman ${ }^{\dagger}$, Stanford Linear Accelerator Center, Menlo Park, CA 94025
}

\section{Abstract}

The present two B-Factories, PEP-II at SLAC in California and KEKB at KEK in Tsukuba, Japan, operate at the Upsilon $4 \mathrm{~S}$ and have reached parameter levels unprecedented for e+e- colliders. They have provided very large data samples for their respective particle detectors, BaBar and BELLE. Luminosity has exceeded $1.5 \times 10^{34} / \mathrm{cm}^{2} / \mathrm{s}$. Beam currents have reached $2.5 \mathrm{~A}$ with 1600 positron bunches spaced by 4 nsec. Continuous injection with the detectors taking data has added significantly to data collection rates by about $40 \%$. Bunch-by-bunch feedback systems damp strong longitudinal and transverse coupled bunch instabilities. The beam-beam interaction has allowed high tune shift levels even in the presence of parasitic crossing and crossing angle effects. Both B-Factory colliders have significant near term luminosity improvement programs.

\section{PARAMETERS}

PEP-II [1] is an $\mathrm{e}^{+} \mathrm{e}^{-}$asymmetric B-Factory Collider located at SLAC operating at $3.1 \mathrm{GeV} \times 9 \mathrm{GeV}$. PEP-II is shown in Figure 1 and 2 and its operating parameters are shown in Table 1. It has reached a luminosity of $9.21 \times 10^{33} / \mathrm{cm}^{2} / \mathrm{s}$ and has delivered an integrated luminosity of $710 \mathrm{pb}^{-1}$ in one day. PEP-II operates in continuous injection mode for both beams boosting the integrated luminosity. The peak positron current has reached $2.45 \mathrm{~A}$ in 1588 bunches. Steady progress is being made in reaching higher luminosity. The progress in luminosity has come from correcting the orbits, lowering $\beta_{\mathrm{y}}{ }^{*}$, moving the fractional horizontal tunes in both rings to just above the half integer $(<0.52)$, and trickle injection of both beams. The goal over the next several years is to reach a luminosity of $2.1 \times 10^{34} / \mathrm{cm}^{2} / \mathrm{s}$. The accelerator physics issues being addressed in PEP-II to reach this goal include the electron cloud instability, beam-beam effects, parasitic beam-beam effects, high RF beam loading [4], shorter bunches, lower $\beta_{\mathrm{y}}{ }^{*}$ interaction region operation, and coupling control. PEP-II has delivered an integrated luminosity to the BaBar detector of over $17 \mathrm{fb}^{-1}$ in one month and more than $264 \mathrm{fb}^{-1}$ since 1999.

KEKB [2] is an $\mathrm{e}^{+} \mathrm{e}^{-}$asymmetric B-Factory Collider located at the KEK laboratory in Tsukuba, Japan, operating at $3.5 \mathrm{GeV} \times 8 \mathrm{GeV}$. KEKB is shown in Figure

Supported by US DOE contracts DE-AC02-76SF00515.
3 and 4 and its operating parameters are shown in Table 2 . It has reached a luminosity of $15.6 \times 10^{33} / \mathrm{cm}^{2} / \mathrm{s}$ and has delivered an integrated luminosity of $1116 \mathrm{pb}^{-1}$ in one day. KEKB operates in continuous injection mode for both beams boosting the integrated luminosity. The peak positron current has reached $1.76 \mathrm{~A}$ in 1389 bunches. Steady progress is being made in reaching higher luminosity. The goal over the next several years is to reach a luminosity of $3.0 \times 10^{34} / \mathrm{cm}^{2} / \mathrm{s}$. The accelerator physics issues being addressed in KEKB to reach this goal include the electron cloud instability, primary beam-beam effects, and crab crossing cavities. KEKB has delivered, over the past six years, an integrated luminosity to the BELLE detector of over $26.5 \mathrm{fb}-1$ in one month and more than $420 \mathrm{fb}^{-1}$ since 1999 .

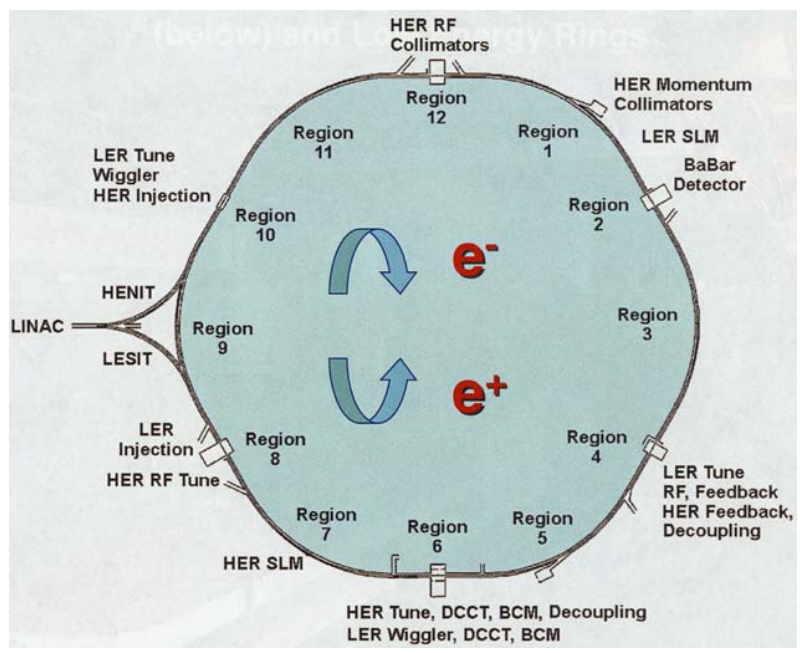

Figure 1. Overview of the PEP-II B-Factory.

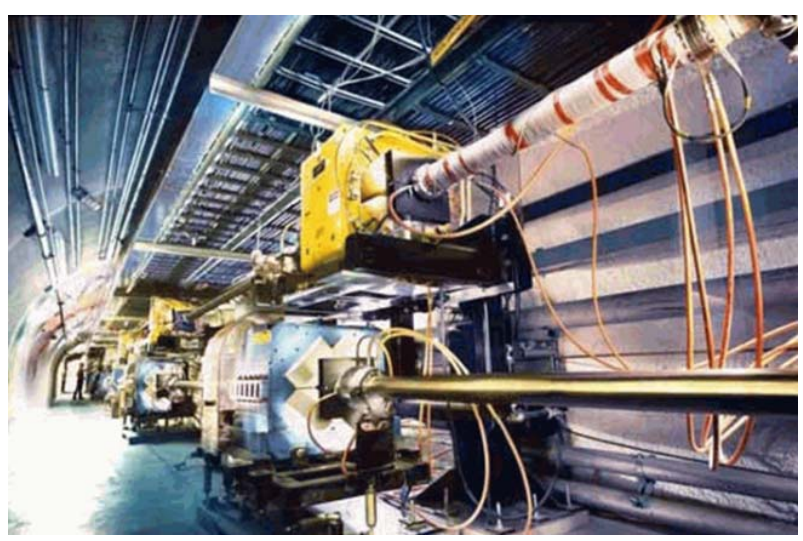

Figure 2. Tunnel view of PEP-II 


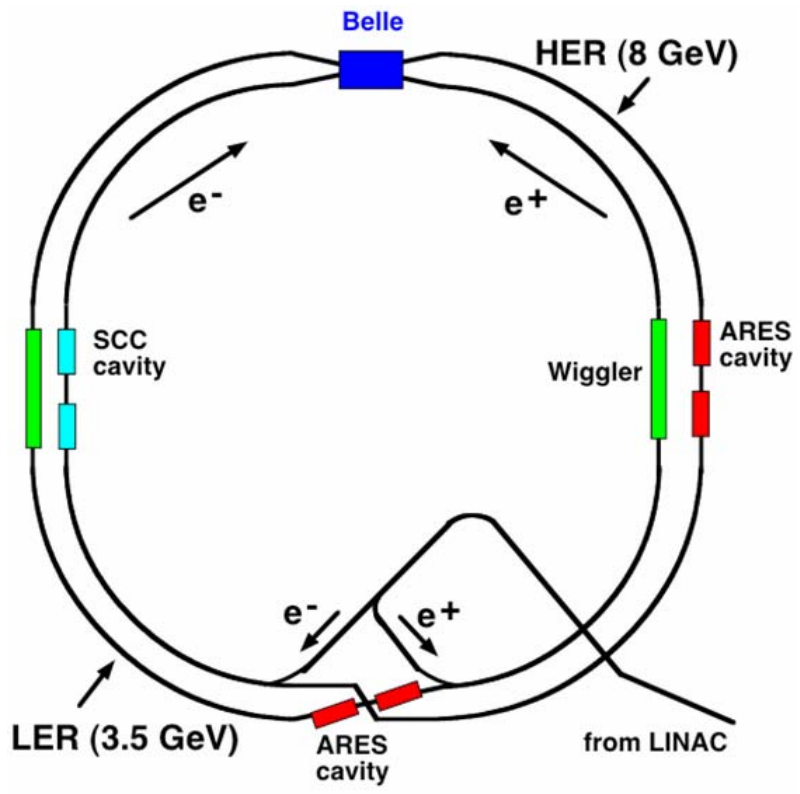

Figure 3 Overview of the KEKB B-Factory

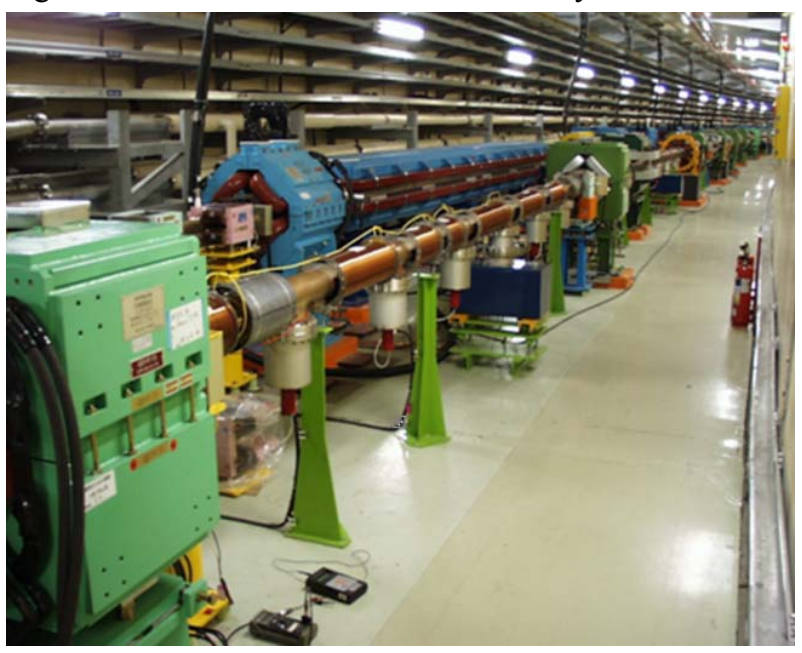

Figure 4 Tunnel view of KEKB

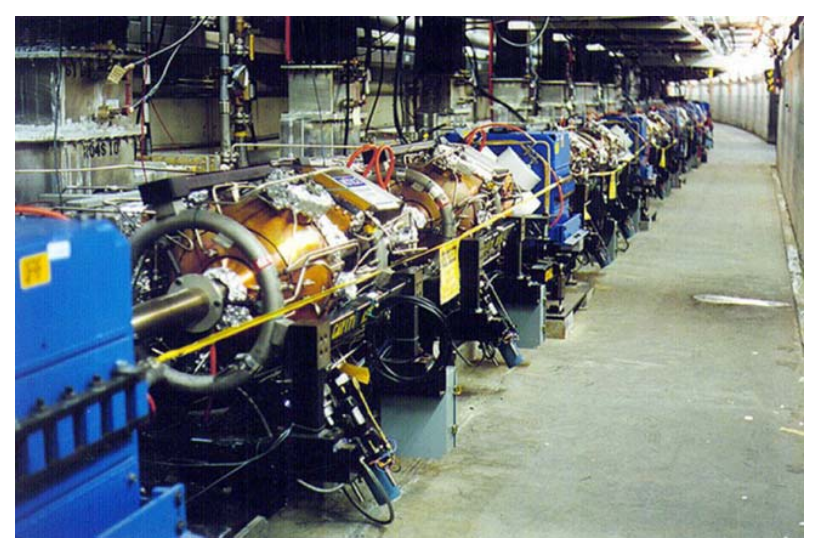

Figure 5 PEP-II RF Cavities
Table 1: PEP-II May 2005 Parameters 3.1 x 9 GeV.

\begin{tabular}{|l|l|l|}
\hline Parameter & $\begin{array}{l}\text { PEP-II } \\
\text { Design }\end{array}$ & $\begin{array}{l}\text { PEP-II } \\
\text { Present }\end{array}$ \\
\hline HER Vertical tune & 23.64 & 23.622 \\
\hline HER Horizontal tune & 24.62 & 24.520 \\
\hline LER Vertical tune & 36.64 & 36.564 \\
\hline LER Horizontal tune & 38.57 & 38.512 \\
\hline HER current $(\mathrm{mA})$ & 750 & 1550 \\
\hline LER current $(\mathrm{mA})$ & 2140 & 2450 \\
\hline Number of bunches & 1658 & 1588 \\
\hline Ion gap $(\%)$ & 5 & 1.8 \\
\hline HER RF klystron/cav & $5 / 20$ & $9 / 26$ \\
\hline HER RF volts $(\mathrm{MV})$ & 14.0 & 15.5 \\
\hline LER RF klystron/cav. & $2 / 4$ & $4 / 8$ \\
\hline LER RF volts $(\mathrm{MV})$ & 3.4 & 4.04 \\
\hline$\beta_{\mathrm{y}}{ }^{*}(\mathrm{~mm})$ & $15-25$ & 11 \\
\hline$\beta_{\mathrm{x}}{ }^{*}(\mathrm{~cm})$ & 50 & $35-49$ \\
\hline Emittance $(\mathrm{x} / \mathrm{y})(\mathrm{nm})$ & $49 / 2$ & $31-59 / 1.4$ \\
\hline$\sigma_{\mathrm{z}}(\mathrm{mm})$ & 11 & $11-12$ \\
\hline Lum hourglass factor & 0.9 & 0.84 \\
\hline Crossing angle $(\mathrm{mrad})$ & 0 & $<0.1$ \\
\hline IP Horiz. size $\Sigma(\mu \mathrm{m})$ & 222 & 170 \\
\hline IP Vert. Size $\Sigma(\mu \mathrm{m})$ & 6.7 & 7.3 \\
\hline HER Horizontal $\xi_{\mathrm{x}}$ & 0.03 & 0.055 \\
\hline HER Vertical $\xi_{\mathrm{y}}$ & 0.03 & 0.046 \\
\hline LER Horizontal $\xi_{\mathrm{x}}$ & 0.03 & 0.053 \\
\hline LER Vertical $\xi_{\mathrm{y}}$ & 0.03 & 0.064 \\
\hline Lumin. $\left(\mathrm{x} 10^{33} / \mathrm{cm}^{2} / \mathrm{s}\right)$ & 3.00 & 9.21 \\
\hline Int. Lum/month $\left(\mathrm{fb}{ }^{-1}\right)$ & 3.3 & 17.04 \\
\hline Total Int. Lum. $\left(\mathrm{fb}{ }^{-1}\right)$ & $30 / \mathrm{year}$ & $>262$ total \\
\hline & & \\
\hline
\end{tabular}

\section{RF SYSTEMS}

The RF systems for PEP-II and KEKB are shown in Figures 5 through 7. PEP has only copper cavities. KEKB has copper cavities for the LER and copper and superconducting cavities for the HER. A world record for for e- current in SC cavities goes to KEKB HER at 1.24 A. The overall world record for (positron) beam current for any RF cavity goes to PEP-II LER at $2.45 \mathrm{~A}$. 
Table 2: KEKB May 2005 Parameters 3.5 x 8 GeV.

\begin{tabular}{|l|l|l|}
\hline Parameter & $\begin{array}{l}\text { KEKB } \\
\text { Design }\end{array}$ & $\begin{array}{l}\text { KEKB } \\
\text { Present }\end{array}$ \\
\hline HER Vertical tune & 43.08 & 41.58 \\
\hline HER Horizontal tune & 47.52 & 44.51 \\
\hline LER Vertical tune & 45.08 & 43.55 \\
\hline LER Horizontal tune & 45.52 & 45.51 \\
\hline HER current $(\mathrm{mA})$ & 1100 & 1242 \\
\hline LER current $(\mathrm{mA})$ & 2600 & 1763 \\
\hline Number of bunches & 5000 & 1389 \\
\hline Ion gap $(\%)$ & 2.3 & 2.3 \\
\hline HER RF klystron/cav & $18 / 36$ & $18 / 18$ \\
\hline HER RF volts $(\mathrm{MV})$ & 20 & 15 \\
\hline LER RF klystron/cav & $10 / 10$ & $10 / 20$ \\
\hline LER RF volts $(\mathrm{MV})$ & 5 & 8 \\
\hline$\beta_{\mathrm{y}}{ }^{*}(\mathrm{~mm})$ & 10 & $5.4-6.2$ \\
\hline$\beta_{\mathrm{x}}{ }^{*}(\mathrm{~cm})$ & 33 & $56-59$ \\
\hline Emittance $(\mathrm{x} / \mathrm{y})(\mathrm{nm})$ & $18 / 0.36$ & $18-24 / 0.4$ \\
\hline$\sigma_{\mathrm{z}}(\mathrm{mm})$ & 4.0 & 6 \\
\hline Hourglass factors & $\sim 0.75$ & $\sim 0.85$ \\
\hline Crossing angle $(\mathrm{mrad})$ & $+/-11$ & $+/-11$ \\
\hline IP Horiz. size $\Sigma(\mu \mathrm{m})$ & 109 & 152 \\
\hline IP Vert. Size $\Sigma(\mu \mathrm{m})$ & 2.7 & 3.0 \\
\hline HER Horizontal $\xi_{\mathrm{x}}$ & 0.039 & 0.074 \\
\hline HER Vertical $\xi_{\mathrm{y}}$ & 0.052 & 0.056 \\
\hline LER Horizontal $\xi_{\mathrm{x}}$ & 0.039 & 0.118 \\
\hline LER Vertical $\xi_{\mathrm{y}}$ & 0.052 & 0.081 \\
\hline Lumin. $\left(\mathrm{x} 10^{33} / \mathrm{cm}^{2} / \mathrm{s}\right)$ & 10.0 & 15.6 \\
\hline Int. Lum/month $\left(\mathrm{fb}{ }^{-1}\right)$ & 10 & 26.5 \\
\hline Total Int. Lum. $\left(\mathrm{fb}{ }^{-1}\right)$ & $100 / \mathrm{year}$ & $>425$ total \\
\hline
\end{tabular}

\section{INTERACTION REGIONS}

The PEP-II interaction region is shown in Figure 8 and the KEKB interaction region in Figure 9. PEP-II uses permanent magnets inside BaBar to allow the beams to collide head on. The KEKB interaction region allows the beams to travel straight into the collision point but then bend going out. The KEKB layout allows for lower backgrounds but with potentially more difficult beambeam effects with a crossing angle of $+/-11 \mathrm{mrad}$.

\section{HALF INTEGER TUNES}

Both PEP-II and KEKB operate with the horizontal tunes just above the half integer $(\sim 0.505-0.515)$ to take advantage of strong dynamic beta effects with current. This enhances the beam-beam tune shifts available. The vertical tunes are nearby at about $(0.58-0.60)$.

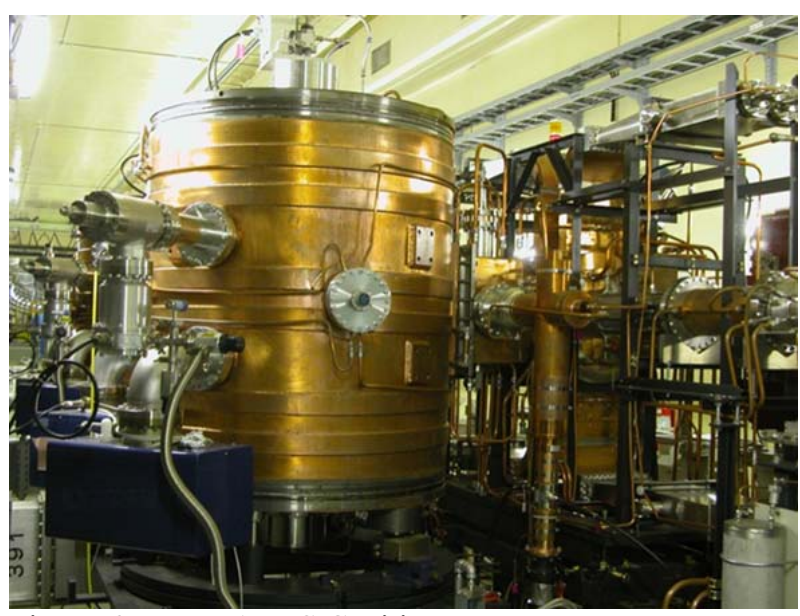

Figure 6 KEKB ARES Cavities

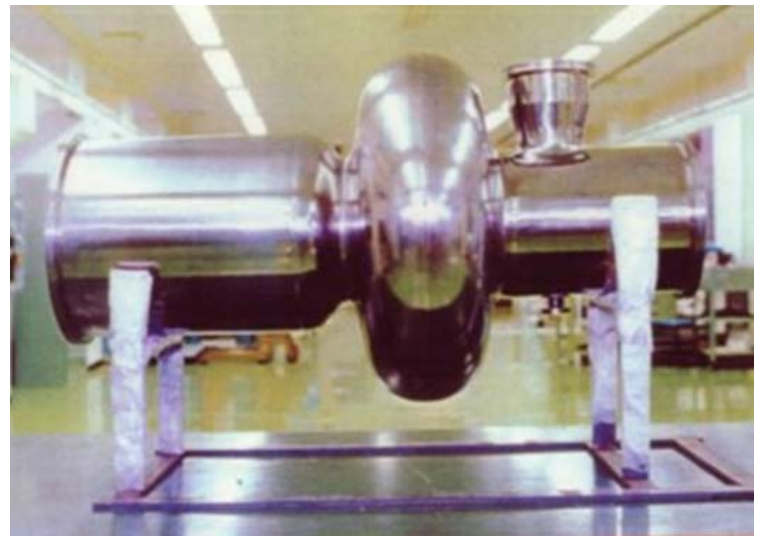

Figure 7 KEKB Superconducting Cavity

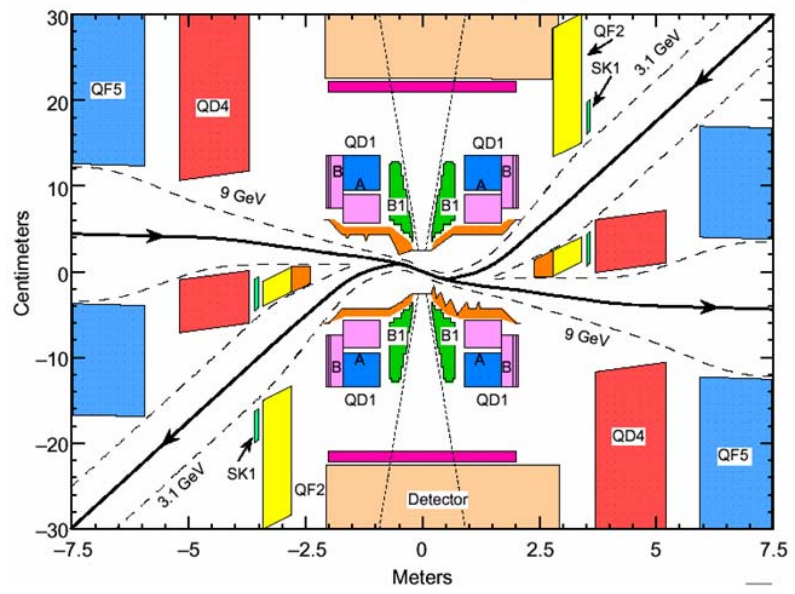

Figure 8 PEP-II Interaction Region 


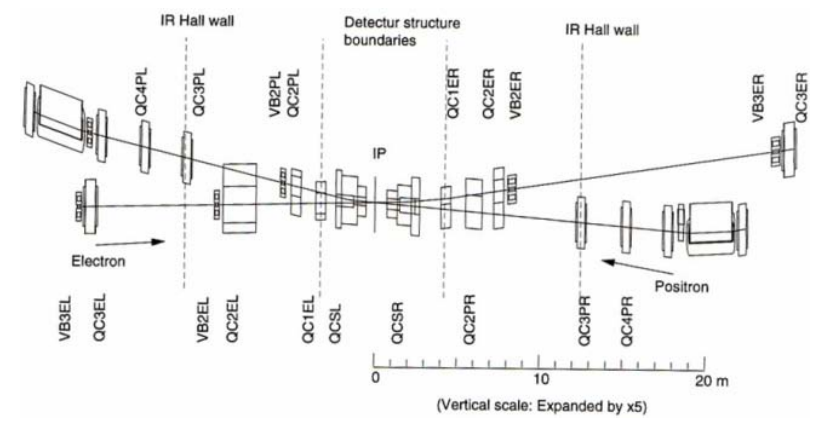

Figure 9 KEKB Interaction Region

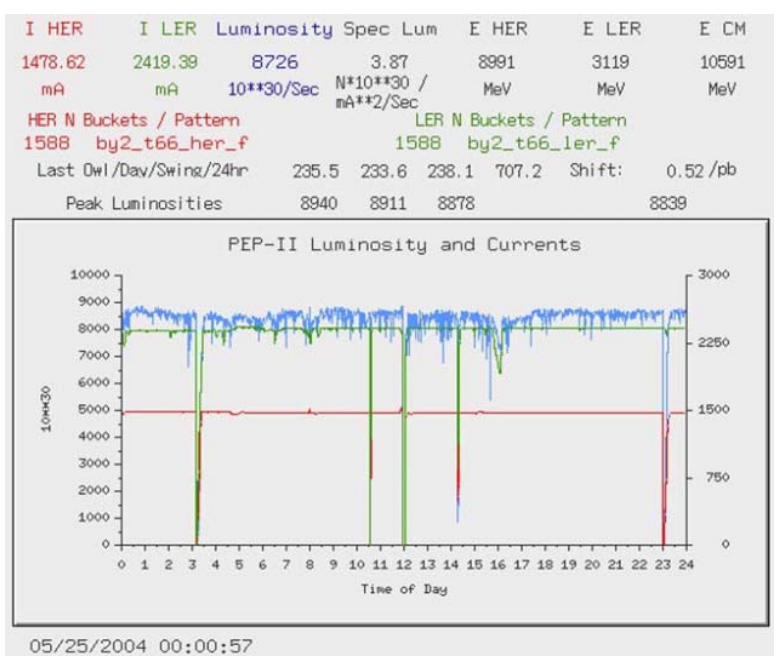

Figure 10 PEP-II trickle charge operation with constant currents and luminosity.

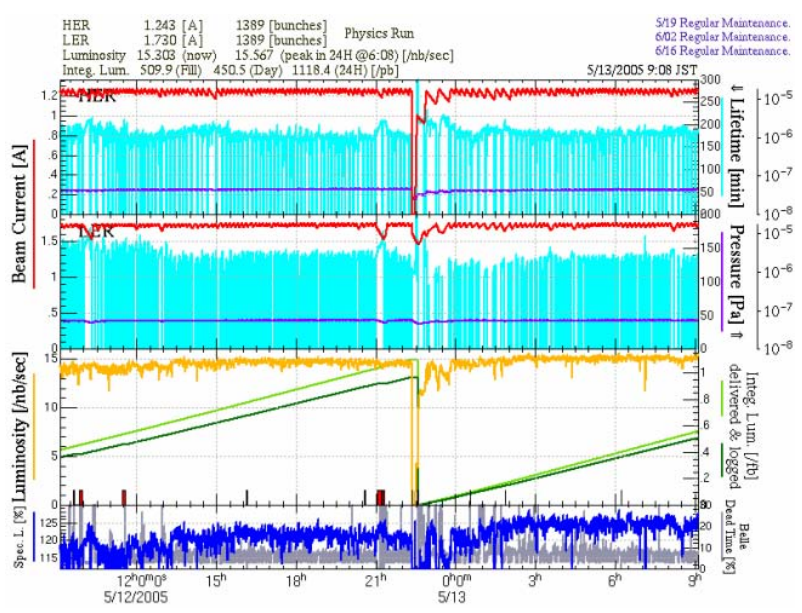

Figure 11 KEKB continuous injection operation.

\section{CONTINUOUS (TRICKLE) INJECTION}

Continuous injection [3] is an operational mode where the beams are topped off while the particle physics detector is running. Continuous injection for the LER in PEP-II was

made to work with BaBar in November 2003. The continuous injection mode for the PEP-II HER was made to work in March 2004. PEP-II can inject with either beam type pulse-by-pulse producing very steady currents and steady luminosity. The injection rate is a few $\mathrm{Hz}$. KEKB went to continuous injection for both rings in early 2004. The KEKB injector must switch back and forth between injection particle types about every 10 minutes. See Figures 10 and 11 for the two accelerators. The production rate for overall integrated luminosity improved by about 30 to $40 \%$ for both colliders.

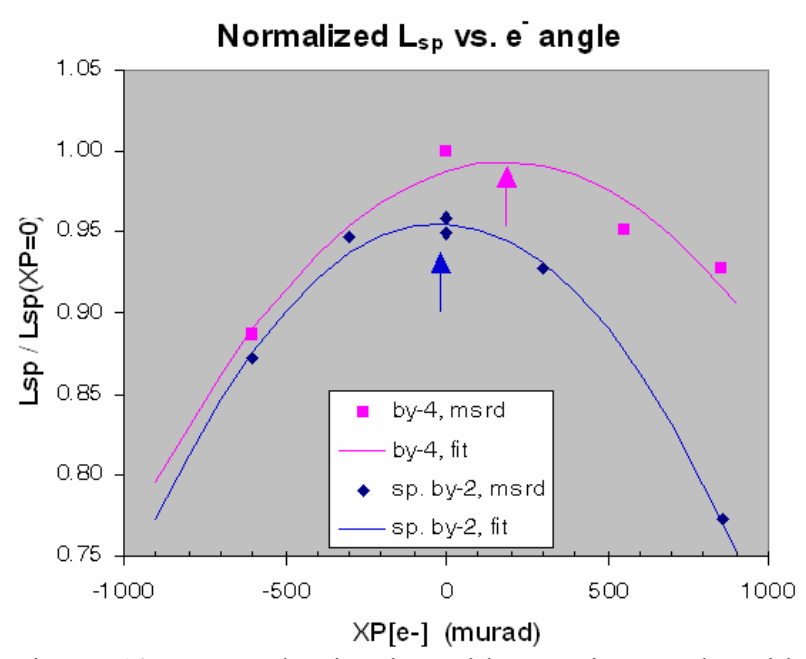

Figure 12 PEP-II luminosity with crossing angle with (lower) and without (upper) parasitic collisions at $0.63 \mathrm{~m}$.

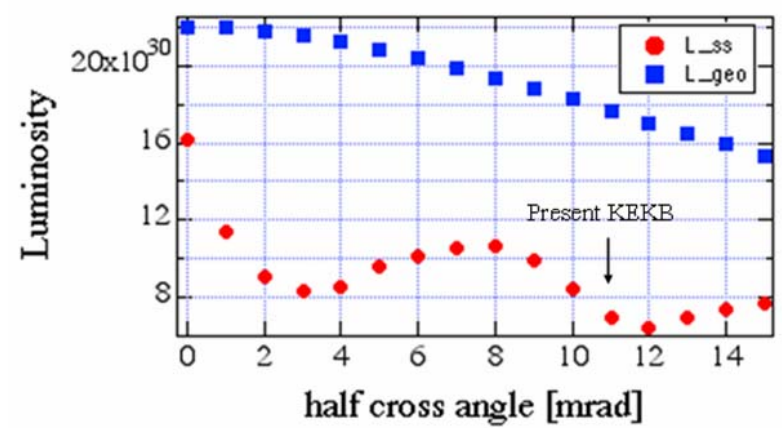

Figure 13 KEKB simulation of luminosity versus crossing angle indicating crab cavities will help the peak luminosity. Geometrical effects are shown above and strong beam-beam effects below [Ohmi, Tawada]

\section{BEAM-BEAM INTERACTION}

The beam-beam parameters have reached large values for both accelerators as can be seen in the two tables. These values are attributed to operation near the horizontal half integer, reduced damping times, and coupling and dispersion correction at the interaction point (IP). Parasitic collisions in PEP-II at $0.63 \mathrm{~m}$ from the IP reduce the peak luminosity about $5 \%$ as seen in Figure 12. $[5,6]$ The crossing angle in KEKB reduces the peak luminosity by about a factor of two as shown in the simulations in Figure 13. The plot indicates that adding crab cavities in KEKB may give a factor of two increase in luminosity. 


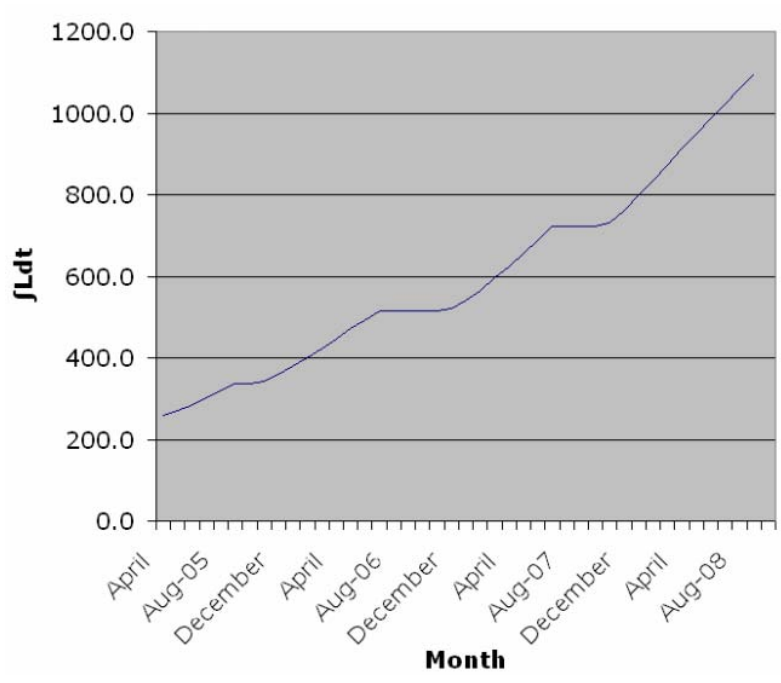

Figure 14. PEP-II projected integrated luminosity to 2008.

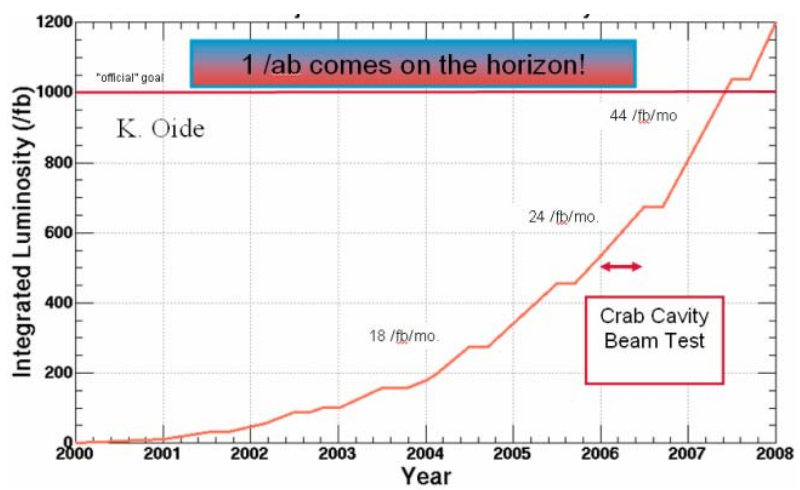

Figure 15. KEKB projected integrated luminosity to 2008.

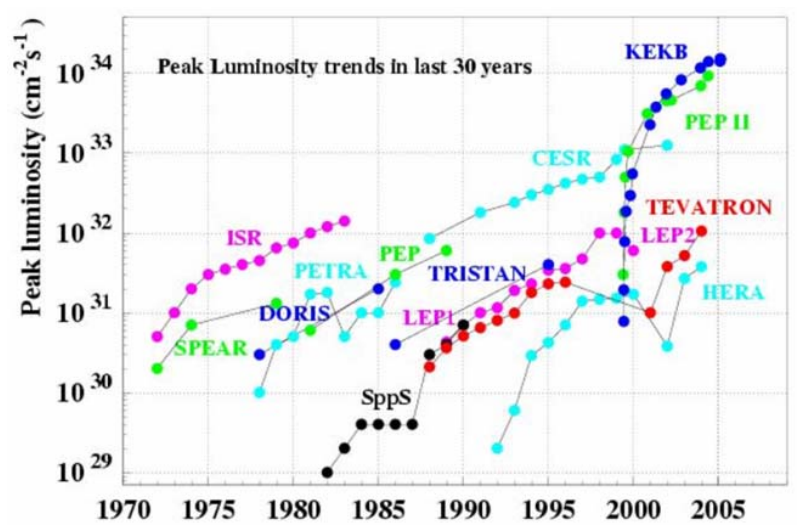

Figure 16. Peak luminosity versus year for various colliders. PEP-II and KEKB have produced a significant step. (K. Oide et al.)

\section{FUTURE PLANS}

Combining the equations for luminosity and the vertical beam-beam parameter, one derives the traditional luminosity parameterization:

$$
L=2.17 \times 10^{34}(1+r) \xi_{y}\left(\frac{E I}{\beta_{y}^{*}}\right) \mathrm{cm}^{-2} \mathrm{sec}^{-1}
$$

with $\mathrm{r}$ the $\mathrm{y}$ to $\mathrm{x}$ aspect ratio $(\sim 0.03)$, E the beam energy, I the beam current, $\xi_{\mathrm{y}}$ the vertical tune shift, and $\beta_{\mathrm{y}}{ }^{*}$ the vertical beta at the collision point.

Over the next two years in PEP-II the currents will be raised about a factor of 1.5 to 2 , the tune shifts increased about $10 \%$ and $\beta_{\mathrm{y}}{ }^{*}$ reduced from $11 \mathrm{~mm}$ to about $8.5 \mathrm{~mm}$. The number of RF stations in the LER will be increased from four to five in order in achieve about $4.4 \mathrm{~A}$. The number of RF stations in the HER will be increased from nine to ten allowing a current of $2.2 \mathrm{~A}$. To shorten the bunch length to reduce the hourglass effects, a lower alpha lattice will be used in HER and a higher RF voltage. The peak luminosity should reach $2.1 \times 10^{34} / \mathrm{cm}^{2} / \mathrm{s}$ in 2007 .

In January 2006 transverse (crab) cavities will be installed in KEKB to gain the luminosity indicated in Figure 13 giving hopefully a factor of two through $\xi_{\mathrm{y}}$. This project upgrade is very interesting as this process has been studied for several decades and will be tested soon.

The expected integrated luminosities for PEP-II and KEKB until 2008 are shown in Figures 14 and 15, respectively. There is a good chance that both accelerators will exceed $1 \mathrm{ab}^{-1}$ in 2008 .

Figure 16 shows the yearly luminosity levels over the lifetimes of many collider projects. PEP-II and KEKB compare very favorably with the historical peak luminosities of similar machines and have set a new high standard by an order of magnitude. Future, order of magnitude, increases in luminosity could be obtained by upgrading these colliders to Super B-Factories [7, 8].

\section{ACKNOWLEDGMENTS}

The author wishes to thank his colleagues at PEP-II and KEKB for providing materials and insights, especially, S. Ecklund, W. Kozanecki, S. Kurokawa, K. Ohmi, K. Oide, D. Rice, M. Sullivan, and U. Wienands.

\section{REFERENCES}

[1] J. Seeman et al., "Performance of the PEP-II BFactory Collider at SLAC," PAC 2005 proceedings.

[2] K. Oide et al., "Performance Status of the KEKB BFactory," PAC 2005 proceedings.

[3] U. Wienands et al., "Lepton Collider Operation with Constant Currents," PAC 2005 proceedings.

[4] A. Novokhatski, "HOM Calculations for Electron Storage Rings," PAC 2005 proceedings.

[5] Y. Cai, K. Ohmi, et al., "Simulations and Experiments of Beam-Beam Effects in e+e- Storage Rings," PAC 2005 proceedings.

[6] W. Kozanecki et al., "Beam-Beam Crossing Angle Measurements at PEP-II," PAC 2005 proceedings.

[7] K. Oide et al., "Super KEKB B-Factory Parameters," PAC 2005 proceedings.

[8] J. Seeman et al, "Parameters of a Super B-Factory Design," PAC 2005 proceedings. 